\title{
Psychosocial correlates of patient-provider family planning discussions among HIV-infected pregnant women in South Africa
}

This article was published in the following Dove Press journal:

Open Access Journal of Contraception

3 April 2017

Number of times this article has been viewed

\author{
Violeta J Rodriguez' \\ Ryan R Cook' \\ Stephen MWeiss' \\ Karl Peltzer ${ }^{2-4}$ \\ Deborah L Jones' \\ 'Department of Psychiatry and \\ Behavioral Sciences, University of \\ Miami Miller School of Medicine, \\ Miami, FL, USA; ${ }^{2}$ HIV/AIDS/STIs and \\ TB (HAST) Research Programme, \\ Human Sciences Research Council, \\ Pretoria, South Africa; ${ }^{3}$ ASEAN \\ Institute for Health Development, \\ Mahidol University, Salaya, Thailand; \\ ${ }^{4}$ Department of Psychology, University \\ of Limpopo, Turfloop, South Africa
}

Correspondence: Deborah L Jones Department of Psychiatry and Behavioral Sciences, University of Miami Miller School of Medicine, 1400 NW 10th Avenue, Miami, FL 33I36, USA

Tel + I 305243204 I

Email d.jones3@med.miami.edu

\begin{abstract}
Patient-provider family planning discussions and preconception counseling can reduce maternal and neonatal risks by increasing adherence to provider recommendations and antiretroviral medication. However, HIV-infected women may not discuss reproductive intentions with providers due to anticipation of negative reactions and stigma. This study aimed to identify correlates of patient-provider family planning discussions among HIV-infected women in rural South Africa, an area with high rates of antenatal HIV and suboptimal rates of prevention of mother-to-child transmission (PMTCT) of HIV. Participants were N=673 pregnant HIV-infected women who completed measures of family planning discussions and knowledge, depression, stigma, intimate partner violence, and male involvement. Participants were, on average, $28 \pm 6$ years old, and half of them had completed at least 10-11 years of education. Most women were unemployed and had a monthly income of less than $\sim$ US\$76. Fewer than half of the women reported having family planning discussions with providers. Correlates of patient-provider family planning discussions included younger age, discussions about PMTCT of HIV, male involvement, and decreased stigma $(p<0.05)$. Depression was indirectly associated with patient-provider family planning discussions through male involvement $(b=-0.010$, bias-corrected $95 \%$ confidence interval $[\mathrm{bCI}][-0.019,-0.005])$. That is, depression decreased male involvement, and in turn, male involvement increased patient-provider family planning discussions. Therefore, by decreasing male involvement, depression indirectly decreased family planning discussions. Study findings point to the importance of family planning strategies that address depression and facilitate male involvement to enhance communication between patients and providers and optimize maternal and neonatal health outcomes. This study underscores the need for longitudinal assessment of men's impact on family planning discussions both pre- and postpartum. Increasing support for provision of mental health services during pregnancy is merited to ensure the health of pregnant women living with HIV and their infants.
\end{abstract}

Keywords: family planning, women, HIV, South Africa, pregnancy, contraception

\section{Introduction}

Preventing unintended pregnancies and increasing family planning are an essential element in the prevention of mother-to-child transmission (PMTCT) of HIV. ${ }^{1}$ South African PMTCT guidelines advise that pregnant HIV-infected women should be counseled on family planning, including postnatal contraception, in clinical consultations. ${ }^{2}$ Preconception counseling and family planning discussions between HIV-infected women and health care providers can improve maternal and neonatal health outcomes by increasing adherence to preconception health care recommendations, e.g., vitamins, nutrition, ${ }^{3}$ and antiretroviral medications for HIV treatment or prophylaxis. ${ }^{4-6}$ However, women in South Africa often have short inter-pregnancy intervals ${ }^{7}$ and may 
access antenatal health care infrequently, ${ }^{8}$ suggesting that health care visits during a current pregnancy may represent the only opportunity available to providers to discuss family planning with women before a subsequent pregnancy. Pregnancy rates within 1 year of pregnancy do not differ between women who intend to have children and those who do not, ${ }^{9}$ and antenatal counseling has been found to increase postnatal contraceptive intentions. ${ }^{10}$ As such, antenatal family planning appears necessary when attending clinical consultations during a current pregnancy, even when women do not express a desire to conceive.

Despite the need to increase family planning and contraception education in support of PMTCT goals, HIV-infected women in South Africa are generally less likely to share reproductive intentions with providers than HIV-negative women and may delay seeking provider guidance until already pregnant. ${ }^{11-13}$ This failure to disclose reproductive intentions to health care staff decreases the likelihood of addressing family planning in clinical consultations. Lack of trust in providers may arise from concerns regarding limited confidentiality and continuity of care in the clinic setting. ${ }^{5}$ Internalized stigma and anticipation of negative reactions from health care providers, such as promotion of abortion $^{14}$ and discouraging women from having children, ${ }^{13}$ may also contribute to reluctance to discuss reproductive intentions. $^{12-15}$

Male partners play an important role in reproductive decision-making and may be a source of emotional support for women during and after pregnancy. ${ }^{16,17}$ However, men may also be a source of distress, ${ }^{16}$ and depression may negatively affect both interpersonal relationships ${ }^{18}$ and the capacity for future-oriented thinking, ${ }^{19}$ decreasing the likelihood of family planning. As such, women experiencing both depression and relationship stress ${ }^{18,20}$ may be less likely to consider future pregnancies and to involve their partners in the process of pregnancy, diminishing the potentially beneficial impact of male involvement in reproductive care. ${ }^{21}$ Similarly, violence against women in intimate relationships (intimate partner violence [IPV]) can further limit women's reproductive autonomy, decreasing the willingness to address reproductive issues with providers as well as adherence to treatment and participation in antenatal care..$^{22,23}$

This study was designed to identify psychosocial correlates of patient-provider family planning discussions among HIV-infected pregnant women in rural Mpumalanga Province, South Africa, an area with high HIV antenatal prevalence rates and suboptimal rates of PMTCT of HIV, ${ }^{24,25}$ and to assess the impact of male partner involvement on the relationship between depression and family planning discussions. It was anticipated that the results could provide programmatic guidance addressing family planning discussions in clinical consultations with HIV-infected women and the role of male involvement during pregnancy.

\section{Methods}

\section{Participants and procedures}

Prior to study onset, Institutional Review Board and Research Ethics Committee approval was obtained from the University of Miami Miller School of Medicine, the Human Sciences Research Council, and the Mpumalanga Provincial Government. Cross-sectional data for this manuscript were drawn from a larger, ongoing study, Protect Your Family. The study protocol, including procedures, recruitment, and sample size determination, has been previously described in detail. ${ }^{26}$ Participants $(\mathrm{N}=673)$ were recruited from 12 rural antenatal clinics (ANCs) in the Gert Sibande and Nkangala districts of Mpumalanga province, South Africa. Eligible candidates were HIV-infected pregnant women, aged 18 years or older, and having a male sexual partner, although male partners were not enrolled. Exclusion criteria included being HIV negative, not being pregnant, and not having a male partner. Women were referred to study staff by ANC nurses and enrolled in the study in a private office after providing verbal and written informed consent in English or local language (isiSesotho, isiZulu). Participants were guided in the use of an Audio Computer-Assisted Self-Interview (ACASI), which presented both written and audio interview questions over headphones in the participant's preferred language (English, isiSesotho, isiZulu) to which the participant responded on a touch screen. ACASI was used to reduce social desirability bias and to facilitate the inclusion of women across all levels of literacy. ${ }^{27}$ To familiarize participants with the ACASI system, the study staff completed the initial demographic questionnaire together with participants; all other assessments included in the ACASI were completed individually, with study staff personnel available nearby to respond to any questions. The interview was $\sim 30$ to 45 minutes in duration, and participants were compensated 50 South African Rand ( US\$5) for time and transportation associated with the assessment.

All study personnel received in-depth training regarding responding to participants self-identifying as depressed or suicidal, and information (leaflets, placards) on suicide and depression was posted in ACASI interview rooms. ${ }^{26} \mathrm{~A}$ trained health care provider was designated and available at all clinics for respondents endorsing severe depression, suicidal ideation, and IPV. Clinic health care providers were 
trained to assess risk and provide triage for further assessment, hospitalization, or outpatient counseling/treatment by licensed psychiatric nurses, social workers, and mental health professionals at associated district hospitals.

\section{Measures}

All measures were translated and back translated into local language and adapted to local settings.

\section{Demographics}

Demographic information included age, education, employment status, income, relationship status, number of children, infant HIV serostatus, and whether the participant had been diagnosed with HIV during pregnancy.

\section{Patient-provider family planning discussions}

Per South African PMTCT protocol, pregnant HIV-infected women are counseled on family planning and strategies to prevent MTCT. ${ }^{2}$ Based on guidelines in the South African PMTCT protocol, ${ }^{2}$ three questions were used to assess patient-provider family planning discussions: "have you talked with a health care provider about planning to get pregnant in the future?", "have you talked with a health care provider about taking medication to avoid transmitting HIV to your baby?", and "do you plan to have more children in the future?". Response options were "Yes/No", and all questions were used as individual items.

\section{Depression}

The Edinburgh Postnatal Depression Scale 10 (EPDS-10) ${ }^{28}$ is a 10-item instrument that was used to evaluate the frequency of symptoms of depression experienced in the past week. Adequate internal consistency has been reported for this scale $(\alpha=0.80),{ }^{29}$ and reliability in this sample was adequate $(a=0.75)$.

\section{Stigma}

The AIDS-Related Stigma Scale (ARSS) ${ }^{30}$ is a nine-item scale that was used to assess stigma, presenting statements representative of HIV stigma, e.g., "People who have AIDS should be ashamed". Higher scores reflected greater levels of internalized, stigma-related attitudes. Adequate internal reliability $\left(\alpha=0.75\right.$ ) for this scale has been reported previously. ${ }^{31}$ The reversed coded item for this scale ("It is safe for people who have AIDS to work with children") was excluded, as the scale demonstrated poor internal reliability $(\alpha=0.58)$ with its inclusion. Excluding the item, reliability was adequate $(\alpha=0.74)$ for this sample.

\section{IPV}

IPV was assessed using an adaptation of the Conflict Tactics Scale 18 (CTS-18), ${ }^{32}$ an 18-item scale that assesses reasoning, psychological IPV, mild physical IPV, and severe physical IPV. For the purposes of this study, only the psychological IPV subscale was used; internal reliability for this subscale was adequate $(\alpha=0.82)$. Nearly all women in this study who report physical IPV report psychological IPV.

\section{Family planning knowledge}

It was believed that women who were more knowledgeable about family planning would be more motivated to discuss family planning with providers. Therefore, family planning knowledge was measured using eight questions that pertained to HIV-affected couples with varied response choices by item; questions were adapted from the Safer Conception Knowledge, Attitudes and Practices (SCKAP) and Family Planning survey. ${ }^{33}$ For each correct response, 1 point was assigned, out of a possible total score of 8 ; the resulting continuous score was utilized (Table 1). As such, greater scores indicated greater knowledge of family planning. Reliability for this scale in this sample was acceptable $(\alpha=0.72)$.

\section{Male involvement}

Male involvement during pregnancy was assessed using a modified version of the Male Involvement Index..29,34 Questions included "Does your male partner attend antenatal care visits with you?", which were then scored as 1 (Yes) or 0 (No), with a range of $0-11$. In this sample, internal consistency for this scale was adequate $(\alpha=0.83)$.

\section{Statistical analyses}

Descriptive statistics were used to analyze demographic and psychosocial variables. To identify psychosocial correlates of family planning discussions between women and providers, bivariate and multivariable analyses were used. To identify bivariate associations, independent samples $t$ tests and chi squares were used to compare women who had discussed family planning with their provider (1) compared to those who had not (0) by demographic and psychosocial characteristics. A non-parametric alternative to the $t$ test, the Mann-Whitney test, was used when distributional assumptions were violated. Given the exploratory nature of this study, all variables found to be associated with family planning discussions at $p<0.15$ on bivariate analyses were included in a subsequent multivariable logistic regression model using backward elimination to identify multivariable associations, which excluded variables not significant at $p<$ 
Table I Family planning knowledge questionnaire and scoring

\section{Family planning knowledge}

Instructions: The following questions are about couples in which one or both members could be HIV positive.

When trying to get pregnant, how much is a man at risk of HIV infection if the woman is positive?

No Chance of infection, $0-10-20-30-40-50-60-70-80-90-$ $100 \%$ will be infected

Score: I point if answer is between 10 and 90

When trying to get pregnant, how much is her partner at risk of HIV infection if a woman is taking HIV medication?

No Chance of infection, $0-10-20-30-40-50-60-70-80-90-$ $100 \%$ will be infected

Score: I point if answer is less than 90

When trying to get pregnant, when in her monthly cycle is a woman the most likely to become pregnant?

I. During her bleeding

2. The weeks in the middle of the cycle

3. The week after the bleeding

4. The week before the bleeding

Score: I point if answer is 'The weeks in the middle of the cycle'

When trying to get pregnant, how can a woman minimize the risk of her partner getting HIV if she is HIV positive?

By only having sexual intercourse without a condom when she is the most fertile

[ ] Yes [ ] No [ ] Do not know

Score: I point if answer is 'Yes'

By taking HIV medications

[ ] Yes [ ] No [ ] Do not know

Score: I point if answer is 'Yes'

When a woman is pregnant, does a man need to use a condom during her pregnancy if she is HIV+?

[ ] Yes [ ] No [ ] Do not know

Score: I point if answer is 'Yes'

After a woman delivers her baby, does a man need to use a condom if she is HIV+?

[ ] Yes [ ] No [ ] Do not know

Score: I point if answer is 'Yes'

If a woman is breastfeeding, does a man need to use a condom if she is HIV+?

[ ] Yes [ ] No [ ] Do not know

Score: I point if answer is 'Yes'

0.15 from the final model. Then, a simple mediation model ${ }^{35}$ was developed, using family planning discussions as the dependent variable, depression as the independent variable, and male involvement as a mediator, while controlling for the variables retained in the reduced multivariable model. Mediation analysis was conducted using the PROCESS macro developed by Hayes for Statistical Package for the Social Sciences (SPSS) (model 4), specifying 5,000 bootstrap samples as recommended by Hayes. ${ }^{33,36}$ A cutoff of $p<0.05$ level was used as the threshold for significance. Results from the mediation analysis are presented using Baron and Kenny's ${ }^{37}$ four-step approach, and the presence of an indirect effect was identified through the absence of zero in the bootstrapped bias-corrected $95 \%$ confidence interval (bCI). ${ }^{36}$ SPSS v22 for Windows was used for all analyses.

\section{Results}

Participants were HIV-infected women between 6 and 30 weeks pregnant $(M=17.8)$, none of whom had attended the Protect Your Family intervention. Of 709 HIV-infected pregnant women asked to participate, eight declined, and 28 had unusable data due to technical difficulties or having participated in some element of the intervention before completion of the baseline assessment, which resulted in a total sample of 673 participants (94.9\% [95\% bCI 93.2, 96.5]). Participants were, on average, $28 \pm 6$ years old. Half of them (50\%) had completed at least 10-11 years of education, and most (78\%) were unemployed. Most participants (68\%) had a monthly income of $<949$ ZAR ( US\$76). Although all women had a partner, less than one-fifth (19\%) reported being married. The majority (79\%) of women had at least one child, and $95 \%$ reported that the serostatus of all of their children was negative. Approximately half of the women were diagnosed with HIV during the current pregnancy. Fewer than half (47\%) of the women reported having family planning discussions with providers; most (85\%) reported discussing MTCT. Further demographic detail and comparisons are presented in Table 2.

\section{Psychosocial correlates of patient- provider family planning discussions}

Engaging in family planning discussions with a provider was associated with discussing HIV transmission to the baby, increased male involvement, greater family planning knowledge, lower levels of stigma, and lower levels of depression and psychological IPV in bivariate analyses. In the reduced multivariable model, family planning discussions were associated with younger age, discussions with provider about MTCT, increased male involvement, and decreased stigma and marginally associated with lower levels of both depression and psychological IPV (Table 3).

\section{Mediation model: depression, male involvement, and family planning discussions}

Variables retained in the reduced multivariable model (age, discussions with provider about MTCT, stigma, and psychological IPV) were included as covariates in the following analyses testing the potential for male involvement to mediate the relationship between depression and patient-provider family planning discussions. In the first model, excluding 
Table 2 Patient-provider family planning discussions by demographic and psychosocial characteristics: bivariate associations ( $\mathrm{N}=673$ )

\begin{tabular}{|c|c|c|c|c|}
\hline Characteristics & All & $\begin{array}{l}\text { Not discussed } \\
(n=359)\end{array}$ & $\begin{array}{l}\text { Discussed } \\
(n=3 \mid 4)\end{array}$ & $\chi^{2} / z / t, p$-Value \\
\hline Age & $28.39(5.73)$ & $28.76(5.65)$ & $27.97(5.80)$ & $-1.71,0.088$ \\
\hline \multicolumn{5}{|l|}{ Educational attainment, years } \\
\hline $0-10$ & $147(2 \mid .8 \%)$ & $71(19.8 \%)$ & $76(24.2 \%)$ & \\
\hline $10-11$ & $334(49.6 \%)$ & $175(48.7 \%)$ & $159(50.6 \%)$ & \\
\hline 12 or more & $192(28.5 \%)$ & $113(31.5 \%)$ & 79 (25.2\%) & $3.97,0.138$ \\
\hline \multicolumn{5}{|l|}{ Employment status } \\
\hline Unemployed & $527(78.3 \%)$ & $276(76.9 \%)$ & $25 I$ (79.9\%) & \\
\hline Employed & $116(17.2 \%)$ & $67(18.7 \%)$ & $49(15.6 \%)$ & \\
\hline Volunteering or student & $30(4.5 \%)$ & $16(4.5 \%)$ & $14(4.5 \%)$ & $\mathrm{I} . \mathrm{II}, 0.575$ \\
\hline \multicolumn{5}{|c|}{ Monthly household income (South African Rand) } \\
\hline$<310(\sim 25)$ & $221(32.8 \%)$ & $117(32.6 \%)$ & $104(33.1 \%)$ & \\
\hline $310-949(-\$ 76)$ & $225(33.9 \%)$ & $124(34.5 \%)$ & $104(33.1 \%)$ & \\
\hline 950 or more & $224(33.3 \%)$ & $118(32.9 \%$ & $106(33.8 \%)$ & $0.15,0.926$ \\
\hline \multicolumn{5}{|l|}{ Marital status } \\
\hline Not married, living separate & $398(59.1 \%)$ & $218(60.7 \%)$ & I $80(57.3 \%)$ & \\
\hline Not married, living together & $150(22.3 \%)$ & 71 (I9.8\%) & 79 (25.2\%) & \\
\hline Married & $125(\mid 8.6 \%)$ & 70 (19.5\%) & 55 (I7.5\%) & $2.86,0.239$ \\
\hline \multicolumn{5}{|l|}{ Number of children } \\
\hline None & $139(20.7 \%)$ & $76(21.2 \%)$ & $63(20.1 \%)$ & \\
\hline One or more & $534(79.3 \%)$ & $283(78.8 \%)$ & $25 I(79.9 \%)$ & $0.123,0.724$ \\
\hline \multicolumn{5}{|l|}{ HIV serostatus of children } \\
\hline Do not know & $506(94.8 \%)$ & 271 (95.8\%) & 235 (93.6\%) & \\
\hline Positive & $28(5.2 \%)$ & $12(4.2 \%)$ & $16(6.4 \%)$ & $1.122,0.269$ \\
\hline \multicolumn{5}{|l|}{ Diagnosed during this pregnancy } \\
\hline No & $308(45.8 \%)$ & $160(44.6 \%)$ & $148(47.1 \%)$ & \\
\hline Yes & $365(54.2 \%)$ & $199(55.4 \%)$ & $166(52.9 \%)$ & $0.44,0.505$ \\
\hline \multicolumn{5}{|c|}{ Has discussed HIV transmission to baby with provider } \\
\hline No & 98 (14.6\%) & 79 (22.0\%) & $19(6.1 \%)$ & $34.27,<0.001$ \\
\hline Yes & 575 (85.4\%) & $280(78.0 \%)$ & 295 (93.9\%) & \\
\hline \multicolumn{5}{|l|}{ Planning to have more children } \\
\hline No & $450(66.9 \%)$ & $247(68.8 \%)$ & $203(64.6 \%)$ & \\
\hline Yes & $223(33.1 \%)$ & $112(31.2 \%)$ & III (35.4\%) & $\mathrm{I} .30,0.254$ \\
\hline Male involvement & $7.10(3.07)$ & $6.42(3.18)$ & $7.87(2.76)$ & $-6.0 \mathrm{I},<0.00 \mathrm{I}^{\mathrm{a}}$ \\
\hline Family planning knowledge & $4.30(1.20)$ & $4.20(1.14)$ & $4.42(1.25)$ & $-3.34,0.00 I^{a}$ \\
\hline Stigma & $0.77(1.36)$ & $0.91(1.44)$ & $0.62(1.25)$ & $-3.93,<0.00 I^{a}$ \\
\hline Depression & $12.03(5.97)$ & $12.62(5.95)$ & $11.36(5.93)$ & $-2.94,0.003^{a}$ \\
\hline Psychological IPV & $3.20(5.3 \mathrm{I})$ & $3.82(5.97)$ & $2.49(4.34)$ & $-3.16,0.002^{a}$ \\
\hline
\end{tabular}

Notes: Data were presented as mean (SD) or $\mathrm{n}(\%)$. ${ }^{2}$ Mann-Whitney test was used for comparison of groups. Bold denotes statistically significant bivariate association with patient-provider family planning discussions.

Abbreviations: SD, standard deviation; IPV, intimate partner violence.

Table 3 Multivariable associations with patient-provider family planning discussions: reduced multivariable analysis ( $\mathrm{N}=673)$

\begin{tabular}{|c|c|c|c|c|c|c|}
\hline Predictors & $b$ & SE & $p$-Value & OR & $\begin{array}{l}\text { Lower } \\
95 \% \mathrm{Cl}\end{array}$ & $\begin{array}{l}\text { Upper } \\
95 \% \text { CI }\end{array}$ \\
\hline Age & -0.034 & 0.015 & 0.022 & 0.967 & 0.940 & 0.995 \\
\hline Discussed transmission of HIV with provider & 1.243 & 0.279 & $<0.001$ & 3.466 & 2.007 & 5.985 \\
\hline Male involvement & 0.131 & 0.029 & $<0.001$ & 1.140 & $\mathrm{I} .077$ & $\mathrm{I} .207$ \\
\hline Stigma & -0.139 & 0.066 & 0.035 & 0.870 & 0.765 & 0.990 \\
\hline Depression & -0.020 & 0.014 & 0.135 & 0.980 & 0.953 & 1.008 \\
\hline Psychological IPV & -0.030 & 0.017 & $0.08 I$ & 0.970 & 0.938 & 1.004 \\
\hline Constant & -0.772 & 0.538 & 0.151 & 0.462 & & \\
\hline
\end{tabular}

Notes: Nagelkerke R squared $=0.150$, Hosmer and Lemeshow test, $\chi^{2}=16.467,0.036$. Bold denotes statistically significant multivariable association with patient-provider family planning discussions.

Abbreviations: SE, standard error; OR, odds ratio; $\mathrm{Cl}$, confidence interval; IPV, intimate partner violence. 
the proposed mediator and after adjusting for covariates, lower depression scores were associated with family planning discussions, $b=-0.029$, odds ratio $(\mathrm{OR})=0.972, p<0.001$. In the second model, depression was associated with reduced male involvement $(b=-0.078, p=0.001)$, accounting for the aforementioned covariates. In the third model, male involvement was associated with family planning discussions $(b=$ $0.131, \mathrm{OR}=1.140, p<0.001)$ after controlling for depression in addition to the other identified covariates. After controlling for male involvement and the covariates previously described, the effect of depression on family planning discussions was no longer significant, $b=-0.020, \mathrm{OR}=0.980, p=0.135$. The indirect effect of depression on patient-provider family planning discussions was statistically significant according to a $95 \% \mathrm{CI}$ for 5,000 bootstrap samples, $b=-0.010$, bCI $[-0.019,-0.005]$. That is, male involvement fully mediated the relationship between depression and family planning discussions, as illustrated in Figure 1.

\section{Discussion}

This study of HIV-infected pregnant women in rural South Africa sought to identify psychosocial correlates of family planning discussions between women and providers and to assess the role of male partner involvement on family planning discussions when women are depressed. Emerging correlates of family planning discussions included younger age, discussions with provider about MTCT, increased male involvement, decreased stigma, lower levels of both depression, and psychological IPV. Decreased male involvement was associated with decreased patient-provider family planning discussions; male involvement fully explained the relationship between depression and family planning discussions. As such, being depressed did not impact family planning discussions directly; rather, depression decreased male involvement. In turn, male involvement increased patient-provider family planning discussions. Therefore, by decreasing male involvement, depression indirectly decreased family planning discussions.

Family planning, including postnatal contraception, can reduce maternal and neonatal risks ${ }^{6,38}$ and is one of the key components of PMTCT protocols. ${ }^{1,2}$ Whereas only half of the women discussed family planning, the majority discussed MTCT with their providers, and discussions regarding MTCT were associated with family planning discussions, both of which support the integration of family planning in the context of PMTCT counseling. Given that women in this study were all pregnant, the high rates of MTCT discussions confirm that women and providers may not address the potential risks of HIV transmission until after women are pregnant, as suggested by previous research. ${ }^{13}$ As many women in rural South Africa access reproductive health care only once during pregnancy, the potential for discussions before they may become pregnant again is limited. ${ }^{8}$ In addition, HIV-infected women are also more likely to miscarry during pregnancy, increasing the likelihood of new pregnancies before women have an opportunity to visit a health care provider. ${ }^{39}$ Mandated health care visits by women during and following pregnancy provide a window of opportunity to discuss family planning. Given the reported limitations to health reproductive care access in Africa, ${ }^{40}$ protocol elements aimed to ensure family planning, which includes postnatal contraceptive counseling, may be better provided during antenatal visits rather than only prior to conception, since antenatal counseling increases postnatal contraceptive intentions. ${ }^{10}$

Consistent with existing literature describing younger women as having a more positive outlook regarding childbearing, ${ }^{41}$ younger women were more likely to discuss family planning with providers. Similarly, in line with existing literature on HIV stigma, ${ }^{14}$ this study found patient-provider family planning discussions to be more likely among women

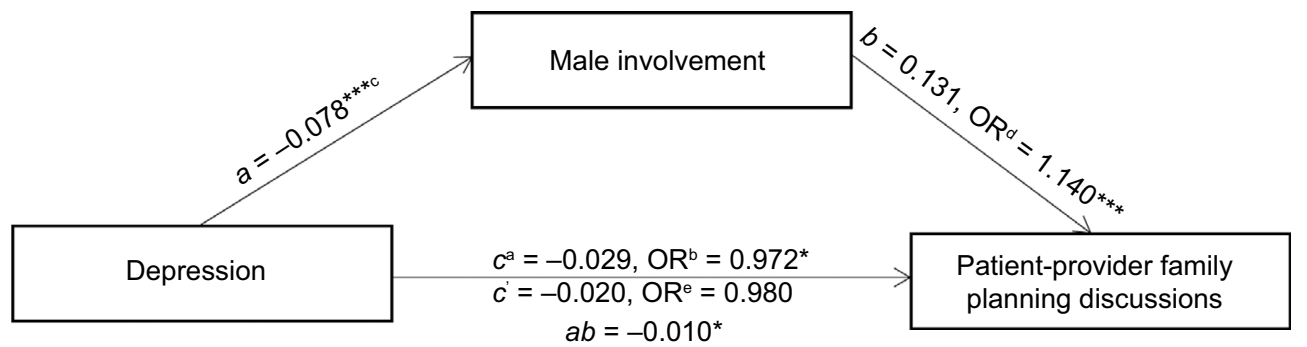

[95\% Cl $-0.02,-0.01]$

Figure I Mediation model: indirect effect of depression on patient-provider family planning discussions through male involvement.

Notes: ${ }^{*} p<0.05 ;{ }^{* *} p<0.001 . c^{\mathrm{a}}$ is the effect of depression on family planning discussions, $c^{\prime}$ is the effect of depression on patient-provider family planning discussions after the effect of male involvement has been accounted for. ${ }^{b, c}$ Adjusted for participant age, discussion with provider about MTCT, stigma, and psychological IPV. ${ }^{\mathrm{d} A d j u s t e d}$ for depression, participant age, discussion with provider about MTCT, stigma, and psychological IPV. eAdjusted for male involvement, depression, participant age, discussion with provider about MTCT, stigma, and psychological IPV.

Abbreviations: MTCT, mother-to-child transmission; IPV, intimate partner violence; OR, odds ratio; $\mathrm{Cl}$, confidence interval. 
reporting less stigma. Interventions aimed at increasing provider- or patient-initiated conception counseling should address women's concerns regarding provider reactions, ${ }^{12-15,42}$ confidentiality of HIV serostatus, and continuity in care. ${ }^{5}$ Training providers in safer conception strategies for HIVinfected women may also enhance clinical practice. ${ }^{43}$ Addressing these concerns may encourage and facilitate an environment conducive to better family planning discussions by helping providers establish a client-centered therapeutic alliance with their patients.

The effect of depression on reproductive health discussions was mediated by male involvement, indicating that although male involvement does increase the rates of engaging in reproductive health discussions, depression was associated with lower rates of male involvement, thereby indirectly decreasing rates of family planning discussions. These results are consistent with previous studies ${ }^{18}$ illustrating that depression may affect interpersonal interactions and result in social rejection, potentially leading to relationship conflict or isolation. ${ }^{20}$ Thus, depression may inhibit male partner involvement and lead to decreased levels of family planning discussions with providers and potentially other positive benefits of male involvement. ${ }^{16,17}$ However, as the cross-sectional design of this study limits causal interpretation, the temporal order of events presented here must be confirmed longitudinally.

When interpreting the results of this study, there are certain limitations to bear in mind. First, causal relationships cannot be drawn because of the study's cross-sectional design. Second, only one item was used to assess family planning discussions. Future studies should address other aspects of family planning discussions with providers that may arise in conjunction with other services or in association with the PMTCT protocol. Third, all of the women in the sample were pregnant, and this manuscript did not assess family planning discussions postnatally. As the rapidly changing antenatal protocol may generate some confusion among HIV-infected women seeking to conceive, ${ }^{26}$ future clinical initiatives should identify alternative opportunities for family planning discussions in clinical consultations to enhance existing pregnancy protocols. The method and frequency of provider-patient communication within clinics should be examined, as well as the potential long-term indirect effect of depression and male involvement on patient-provider family planning discussions.

While the prevalence of MTCT of HIV in South Africa has decreased from $3.5 \%$ in $2010^{24}$ to $2.2 \%$ in 2016 among exclusive breastfeeding mothers on antiretroviral therapy, ${ }^{44}$ $\mathrm{HIV}$ ANC prevalence rates remain higher in some regions of
South Africa. ${ }^{25}$ This study identified factors associated with antenatal patient-provider family planning discussions and highlighted the importance of family planning strategies to counteract depression $^{45}$ and facilitate male involvement as a strategy to enhance communication between patients and providers and optimize maternal and neonatal health outcomes. Evidence to support the contribution of men during pregnancy continues to accumulate, and this study underscores the need for longitudinal assessment of men's impact on family planning discussions both pre- and postpartum. Similarly, increasing support is evident for provision of mental health services during pregnancy to ensure the health of pregnant women living with HIV and their infants.

\section{Acknowledgments}

This study was funded by a grant from the National Institutes of Health (NIH), R01HD078187, and made possible with support from the University of Miami Miller School of Medicine Center for AIDS Research, NIH grant P30AI073961, and from the participating families.

\section{Disclosure}

The authors report no conflicts of interest in this work.

\section{References}

1. Baek C, Rutenberg N. Addressing the Family Planning Needs of HIV-Positive PMTCT Clients: Baseline Findings from An Operations Research Study. Washington, DC: Population Council; 2005.

2. Department of Health: Republic of South Africa. National Consolidated Guidelines for the Prevention of Mother-to-Child Transmission of HIV (PMTCT) and the Management of HIV in Children, Adolescents, and Adults. Available from: http://www.sahivsoc.org/Files/ART\%20Guidelines\%2015052015.pdf. Accessed February 07, 2017.

3. Jean J, Coll A, Monda M, Potter J, Jones D. Perspectives on safer conception practices and preconception counseling among women living with HIV. Health Care Women Int. 2016;37(10):1096-1118.

4. Audet CM, Blevins M, Chire YM, et al. Engagement of men in antenatal care services: increased HIV testing and treatment uptake in a community participatory action program in Mozambique. AIDS Behav. 2016;20(9):2090-2100.

5. Gourlay A, Wringe A, Birdthistle I, Mshana G, Michael D, Urassa M. "It is like that, we didn't understand each other": exploring the influence of patient-provider interactions on prevention of mother-to-child transmission of HIV service use in rural Tanzania. PLoS One. 2014; 9(9):e106325.

6. Steiner RJ, Dariotis JK, Anderson JR, Finocchario-Kessler S. Preconception care for people living with HIV: recommendations for advancing implementation. AIDS. 2013;27(suppl 1):S113-S119.

7. Hung KJ, Scott J, Ricciotti HA, Johnson TR, Tsai AC. Community-level and individual-level influences of intimate partner violence on birth spacing in sub-Saharan Africa. Obstet Gynecol. 2012;119(5):975-982.

8. Myer L, Harrison A. Why do women seek antenatal care late? Perspectives from rural South Africa. J Midwifery Womens Health. 2003; 48(4):268-272.

9. Smith KB, van der Spuy ZM, Cheng L, Elton R, Glasier AF. Is postpartum contraceptive advice given antenatally of value? Contraception. 2002;65(3):237-243. 
10. Keogh SC, Urassa M, Kumogola Y, Kalongoji S, Kimaro D, Zaba B. Postpartum contraception in Northern Tanzania: patterns of use, relationship to antenatal intentions, and impact of antenatal counseling. Stud Fam Plann. 2015;46(4):405-422.

11. Kaida A, Laher F, Strathdee SA, et al. Childbearing intentions of HIV-positive women of reproductive age in Soweto, South Africa: the influence of expanding access to HAART in an HIV hyperendemic setting. Am J Public Health. 2011;101(2):350-358.

12. Kawale P, Mindry D, Phoya A, Jansen P, Hoffman RM. Provider attitudes about childbearing and knowledge of safer conception at two HIV clinics in Malawi. Reprod Health. 2015;12:17.

13. Mindry DL, Crankshaw TL, Maharaj P, et al. "We have to try and have this child before it is too late": missed opportunities in client-provider communication on reproductive intentions of people living with HIV. AIDS Care. 2015;27(1):25-30.

14. Cooper D, Harries J, Myer L, Orner P, Bracken H, Zweigenthal V. "Life is still going on": reproductive intentions among HIV-positive women and men in South Africa. Soc Sci Med. 2007;65(10):2186-2186. (vol 65, p. 274, 2007).

15. Myer L, Morroni C, Rebe K. Prevalence and determinants of fertility intentions of HIV-infected women and men receiving antiretroviral therapy in South Africa. AIDS Patient Care STDS. 2007;21(4):278-285.

16. Jones D, Chakhtoura N, Cook R. Reproductive and maternal healthcare needs of HIV infected women. Curr HIV/AIDS Rep. 2013;10(4): 333-341.

17. Maman S, Moodley D, Groves AK. Defining male support during and after pregnancy from the perspective of HIV-positive and HIVnegative women in Durban, South Africa. J Midwifery Womens Health. 2011;56(4):325-331.

18. Hames JL, Hagan CR, Joiner TE. Interpersonal processes in depression. Annu Rev Clin Psycho. 2013;9:355-377.

19. Bjarehed J, Sarkohi A, Andersson G. Less positive or more negative? Future-directed thinking in mild to moderate depression. Cogn Behav Ther. 2010;39(1):37-45.

20. Coyne JC, Thompson R, Palmer SC. Marital quality, coping with conflict, marital complaints, and affection in couples with a depressed wife. J Fam Psychol. 2002;16(1):26-37.

21. Jones D, Peltzer K, Villar-Loubet O, et al. Reducing the risk of HIV infection during pregnancy among South African women: a randomized controlled trial. AIDS Care. 2013;25(6):702-709.

22. Jewkes RK, Levin JB, Penn-Kekana LA. Gender inequalities, intimate partner violence and HIV preventive practices: findings of a South African cross-sectional study. Soc Sci Med. 2003;56(1):125-134.

23. Hatcher AM, Stockl H, Christofides N, et al. Mechanisms linking intimate partner violence and prevention of mother-to-child transmission of HIV: a qualitative study in South Africa. Soc Sci Med. 2016;168:130-139.

24. Barron P, Pillay Y, Doherty T, et al. Eliminating mother-to-child HIV transmission in South Africa. Bull World Health Organ. 2013;91(1):70-74.

25. Mpumalanga Provincial AIDS Council. Annual Progress Report 2014-2015: Provincial Strategic Plan 2012-2016. Available from: http://sanac.org.za/wp-content/uploads/2016/04/MP_PSP-ANNUALPROGRESS-REPORT_Final-Report.pdf. Accessed February 07, 2017.

26. Jones D, Peltzer K, Weiss SM, et al. Implementing comprehensive prevention of mother-to-child transmission and HIV prevention for South African couples: study protocol for a randomized controlled trial. Trials. 2014;15:417.
27. Metzger DS, Koblin B, Turner C, et al. Randomized controlled trial of audio computer-assisted self-interviewing: utility and acceptability in longitudinal studies. HIVNET Vaccine Preparedness Study Protocol Team. Am J Epidemiol. 2000;152(2):99-106.

28. Cox JL, Holden JM, Sagovsky R. Detection of postnatal depression. Development of the 10-item Edinburgh Postnatal Depression Scale. $\mathrm{Br}$ J Psychiatry. 1987;150:782-786.

29. Peltzer K, Sikwane E, Majaja M. Factors associated with short-course antiretroviral prophylaxis (dual therapy) adherence for PMTCT in Nkangala district, South Africa. Acta Paediatr. 2011;100(9):1253-1257.

30. Kalichman SC, Simbayi LC, Cloete A, Mthembu PP, Mkhonta RN, Ginindza T. Measuring AIDS stigmas in people living with HIV/AIDS: the Internalized AIDS-Related Stigma Scale. AIDS Care. 2009;21(1):87-93.

31. Kalichman SC, Simbayi LC, Jooste S, et al. Development of a brief scale to measure AIDS-related stigma in South Africa. AIDS Behav. 2005;9(2):135-143.

32. Straus M. Measuring intrafamily conflict and violence: the conflict tactics (CT) scales. J Marriage Fam. 1979;41(1):75-88.

33. Idonije B, Oluba O, Otamere H. A study on knowledge, attitude and practice of contraception among secondary school students in Ekpoma, Nigeria. JPCS. 2011;2:22-27.

34. Byamugisha R, Tumwine JK, Semiyaga N, Tylleskar T. Determinants of male involvement in the prevention of mother-to-child transmission of HIV programme in Eastern Uganda: a cross-sectional survey. Reprod Health. 2010;7:12.

35. Preacher KJ, Hayes AF. SPSS and SAS procedures for estimating indirect effects in simple mediation models. Behav Res Methods Instrum Comput. 2004;36(4):717-731.

36. Hayes M. Statistical Digital Signal Processing and Modeling. New York, NY: John Wiley \& Sons; 2009.

37. Baron RM, Kenny DA. The moderator-mediator variable distinction in social psychological research: conceptual, strategic, and statistical considerations. J Pers Soc Psychol. 1986;51(6):1173-1182.

38. Myer L, Rabkin M, Abrams EJ, Rosenfield A, El-Sadr WM; Columbia University MTCT-Plus Initiative. Focus on women: linking HIV care and treatment with reproductive health services in the MTCT-Plus Initiative. Reprod Health Matters. 2005;13(25):136-146.

39. Kim H, Kasonde P, Mwiya M, et al. Pregnancy loss and role of infant HIV status on perinatal mortality among HIV-infected women. BMC Pediatr. 2012;12(1):1.

40. Scorgie F, Nakato D, Harper E, et al. 'We are despised in the hospitals': sex workers' experiences of accessing health care in four African countries. Cult Health Sex. 2013;15(4):450-465.

41. Nduna M, Farlane L. Women Living with HIV in South Africa and their concerns about fertility. AIDS Behav. 2009;13(suppl 1):S62-S65.

42. Schwartz SR, Mehta SH, Taha TE, Rees HV, Venter F, Black V. High pregnancy intentions and missed opportunities for patient-provider communication about fertility in a South African cohort of HIV-positive women on antiretroviral therapy. AIDS Behav. 2012;16(1):69-78.

43. Okoror TA, Belue R, Zungu N, Adam AM, Airhihenbuwa CO. HIV positive women's perceptions of stigma in health care settings in Western Cape, South Africa. Health Care Women Int. 2014;35(1):27-49.

44. Goga AE, Dinh TH, Jackson DJ, et al; South Africa PMTCT Evaluation (SAPMCTE) Team. Population-level effectiveness of PMTCT Option A on early mother-to-child (MTCT) transmission of HIV in South Africa: implications for eliminating MTCT. J Glob Health. 2016;6(2):020405.

45. Ross DA, South A, Weller I, Hakim J. HIV treatment and care systems: the way forward. AIDS. 2012;26(suppl 2):S147-S152. 
Open Access Journal of Contraception is an international, peerreviewed, open access, online journal, publishing original research, reports, reviews and commentaries on all areas of contraception. In addition to clinical research, demographics and health-related aspects, the journal welcomes new findings in animal and preclinical studies relating to understanding the biological mechanisms and practical development of new contraceptive agents. The manuscript management system is completely online and includes a very quick and fair peer-review system. Visit http://www.dovepress.com/testimonials.php to read real quotes from published authors.

Submit your manuscript here: https://www.dovepress.com/open-access-journal-of-contraception-journal 